\title{
Comparison of two rapid tests for anti-phenolic glycolipid-I serology in Brazil and Nepal
}

\author{
Mariane Martins de Araújo Stefani', Adriano Badotti Grassi', Lucas Henrique Sampaio', \\ Ana Lúcia Osório Maroclo de Sousa', Maurício Barcelos Costa', Pauline Scheelbeek², \\ Kapil D Neupane ${ }^{3}$, Deanna A Hagge ${ }^{3}$, Murdo Macdonald ${ }^{3}$, Sang-Nae Cho ${ }^{4}$, \\ Linda Oskam², Samira Bührer-Sékula1/+
}

\footnotetext{
'Instituto de Patologia Tropical e Saúde Pública, Universidade Federal de Goiás, Goiânia, GO, Brasil ${ }^{2}$ KIT Biomedical Research, Amsterdam, The Netherlands ${ }^{3}$ Mycobacterial Research Laboratories, Anandaban Hospital, Kathmandu, Nepal ${ }^{4}$ Department of Microbiology, Institute of Immunology and Immunological Diseases, Yonsei University College of Medicine, Seoul, Republic of Korea
}

The diagnosis of leprosy continues to be based on clinical symptoms and early diagnosis and treatment are critical to preventing disability and transmission. Sensitive and specific laboratory tests are not available for diagnosing leprosy. Despite the limited applicability of anti-phenolic glycolipid-I (PGL-I) serology for diagnosis, it has been suggested as an additional tool to classify leprosy patients (LPs) for treatment purposes. Two formats of rapid tests to detect anti-PGL-I antibodies [ML immunochromatography assay (ICA) and ML Flow] were compared in different groups, multibacillary patients, paucibacillary patients, household contacts and healthy controls in Brazil and Nepal. High ML Flow intra-test concordance was observed and low to moderate agreement between the results of ML ICA and ML Flow tests on the serum of LPs was observed. LPS were "seroclassified" according to the results of these tests and the seroclassification was compared to other currently used classification systems: the World Health Organization operational classification, the bacilloscopic index and the Ridley-Jopling classification. When analysing the usefulness of these tests in the operational classification of PB and MB leprosy for treatment and follow-up purposes, the ML Flow test was the best point-of-care test for subjects in Nepal and despite the need for sample dilution, the ML ICA test yielded better performance among Brazilian subjects. Our results identified possible ways to improve the performance of both tests.

Key words: leprosy - serologic tests - immunochromatography

Leprosy is a chronic infectious disease caused by $M y$ cobacterium leprae. This disease predominantly affects the skin and peripheral nerves and can cause significant nerve destruction, leading to handicaps and/or deformities (Scollard 2008). Leprosy is a spectral disease: the clinical manifestations are dependent on the host immune response (Ridley \& Jopling 1966). At one end of the spectrum, lepromatous leprosy (LL) patients exhibit a higher bacterial index (BI), high titres of M. leprae-specific antibodies and limited or no specific cell-mediated immunity. By contrast, tuberculoid leprosy (TT) patients demonstrate no detectable BI, low or absent titres of $M$. leprae-specific antibodies and significant specific cellmediated immunity (CMI) (Chanteau et al. 1989, Cho et al. 1991, Sampaio et al. 2011). Other borderline forms occupy the spectrum between the humoral-dominated LL form and the CMI-dominated TT form: borderline lepromatous (BL), borderline-borderline (BB) and borderline tuberculoid (BT) (Ridley \& Jopling 1966). According to the $\mathrm{BI}$ of the patients, TT/BT forms are regarded as

Financial support: IDEAL

MM de AS is a recipient of a fellowship from CNPq (310582/2011-3). +Corresponding author: samira@buhrer.net

Received 22 March 2012

Accepted 14 August 2012 paucibacillary $(\mathrm{PB})$ and $\mathrm{BB} / \mathrm{BL} / \mathrm{LL}$ forms are considered multibacillary (MB) disease.

Despite the success of multidrug therapy (MDT) in reducing the number of registered leprosy cases worldwide, approximately 210,000 newly diagnosed patients are still registered every year (WHO 2010). The MDT drug regimen and its duration vary for $\mathrm{PB}$ and MB leprosy. In 1998, the World Health Organization recommended a simplified operational leprosy classification system for PB and MB leprosy based on the number of skin lesions: patients with up to five lesions were designated as PB and patients with six or more lesions were designated as MB. Currently, leprosy diagnosis is still based on clinical symptoms and early diagnosis and treatment are crucial to prevent disability and further transmission (Dockrell et al. 2011). Sensitive and specific laboratory tests are not available for the diagnosis of leprosy. The presence of antibodies against phenolic glycolipid-I (PGL-I), an immunodominant M. lepraespecific antigen, correlates with a higher BI in leprosy patients (LPs) (Douglas \& Worth 1984, Chujor et al. 1991, Bührer-Sékula et al. 2003). ELISA, particle agglutination, dipstick tests and rapid lateral-flow tests have been developed to detect anti-PGL-I IgM antibodies (Brett et al. 1986, Petchclai et al. 1988, Bührer-Sékula et al. 2003). The anti-PGL-I serology reflects the leprosy spectrum and BI positivity: high positive rates are found among MB patients and low positive rates are found among PB 
patients (Moura et al. 2008). Therefore, despite its limited application for the general diagnosis of leprosy, antiPGL-I serology has been suggested as an auxiliary tool to differentiate between PB and MB LPs to prescribe the corresponding MDT regimen. As a point-of-care (POC) test, the anti-PGL-I rapid lateral flow test is easy to perform, does not require refrigeration or other devices and can be implemented in the outpatient routine to aid the classification of patients as PB or MB for treatment purposes. The combination of clinical examination and antiPGL-I testing can lead to a significant reduction in the misclassification of LPs, compared with lesion counting alone (Bührer-Sékula et al. 2003, 2009). The use of a rapid anti-PGL-I test to correctly classify patients can facilitate the prescription of the appropriate MDT regimen and the prevention of transmission.

In 2004, an international research consortium, Initiative for Diagnostic and Epidemiological Assays for Leprosy (IDEAL) was formed and one of its goals was to foster the development of laboratory tests for the diagnosis of leprosy (Dockrell et al. 2011). As part of this initiative, the present study compared two rapid tests to detect anti-PGL-I antibodies against $M$. leprae in field situations in two leprosy endemic countries: Brazil and Nepal. In this study, we have used the ML Flow test, which detects anti-PGL-I IgM antibodies in whole blood and in serum, and the ML- immunochromatography assay (ICA) test, which detects anti-PGL-I IgM, IgG and IgA antibodies in serum. The results of both lateral flow tests were compared in Nepal and Brazil and the agreement between them was reported. The potential applications, strengths and limitations of these classification systems are discussed.

\section{SUBJECTS, MATERIALS AND METHODS}

In this study, the percentage of positive and negative ML Flow and ML ICA tests and the agreement between these tests were compared among four study groups in Brazil and Nepal. Based on the results of these tests, we "seroclassified" LPs as MB leprosy (positive serology) or PB leprosy (negative serology). This seroclassification was compared to other currently used classification systems: the WHO operational classification (based on the number of lesions), the bacilloscopic index and the Ridley and Jopling (1966) classification.

Study groups - The study populations in Brazil and Nepal included four different groups: (i) MB LPs (MB), (ii) PB LPs (PB), (iii) healthy household contacts (HHCs) of MB LPs and (iv) healthy controls from corresponding leprosy endemic control (EC) areas.

Only newly diagnosed and previously untreated patients were eligible for inclusion in the Brazilian leprosy groups. In Nepal, patients were also included in the study within the first three months after starting MDT. Prior to recruitment, all patients were examined by an experienced leprosy clinician. LPs were fully characterised according to the Ridley and Jopling (1966) criteria by taking into account clinical, bacilloscopic and histopathological findings. The disability grade of LPs was also assessed (WHO 1988). When serology data were used as a tool to aid PB and MB classification in field situations, the WHO operational leprosy classification system for PB and MB leprosy was employed (WHO 1998); accordingly, LPs were classified as PB or MB based on the number of skin lesions. The presence of thickened nerves was not considered.

HHC were adults without signs or symptoms of leprosy or tuberculosis (TB) and that had lived in the same household with an untreated MB patient for at least six months. Endemic controls were healthy individuals who had no history of TB or leprosy in the family.

Brazilian LPs were recruited at the main outpatient clinic in central-western Brazil (Reference Center for Diagnostics and Therapeutics, Goiânia, state of Goiás) from February 2007-November 2007. EC were recruited among public college students from the same endemic setting. In Nepal, participants were recruited from the Anandaban Hospital and from Anandaban's weekly satellite clinic at Patan Hospital in Kathmandu between December 2006March 2008. EC were recruited from among Nepalese college and clinical students visiting for training at the Anandaban Hospital or at the satellite clinic.

Test descriptions - Two different lateral flow tests, the ML Flow test and the ML ICA test, were used in this comparative study. The ML Flow test was manufactured by OmegaTeknika Limited (Dublin, Ireland) and by the Royal Tropical Institute (KIT, Amsterdam, Netherlands) according to the specifications defined by BührerSékula et al. (2003). The antigen used in the ML Flow test to detect IgM antibodies was natural trisaccharidephenylpropionyl-bovine serum albumin (NT-P-BSA), a semi-synthetic trisaccharide of the PGL-I antigen linked to BSA. The ML Flow test employs the same device and general protocol for both whole blood and serum samples. In brief, $5 \mu \mathrm{L}$ of serum or blood was deposited in the sample application receptacle and $130 \mu \mathrm{L}$ of running buffer was added.

The ML ICA test was developed and produced in collaboration with Yonsei University and Standard Diagnostics Inc (Suweon, Republic of Korea). The antigens used in the ML ICA test are a semi-synthetic disaccharide of the PGL-I antigen bound to human serum albumin (ND-O-HSA) or ND-O-BSA. The ML ICA test detects IgM, IgG and IgA antibodies. The ML ICA test features two different formats: one for whole blood and one for serum samples. ML ICA tests were performed according to the manufacturer's recommendations. In brief, $15 \mu \mathrm{L}$ of serum or blood was diluted in $135 \mu \mathrm{L}$ of running buffer and $100 \mu \mathrm{L}$ of this dilution was deposited in the corresponding sample application receptacle. ML ICA tests for whole blood did not perform as specified by the manufacturer.

The visual readings for both tests were performed 5-10 min after buffer application. The results were scored according to positivity: 0 and $0.5=$ negative, $1+$, $2+, 3+$ and $4+=$ positive.

Statistical analyses - Descriptive analyses of clinical and epidemiological variables and their frequencies were performed. The agreement between the results of individual tests was calculated by cross-tabulation and results were expressed as percentages with the graduated kappa 
index (к): low (0-0.5), moderate (0.51-0.75) and excellent (0.76-1), following the Svanholm protocol. The correlation between the results was measured by the correlation index (r), in which $r=0$ indicates no correlation and $r=1$ indicates perfect positive correlation. Data analysis was performed with IBM SPSS statistics version 17.

Ethical issues - In Brazil, this study was approved by a local ethics committee (Ethics in Human Research and Animal, Hospital of the Federal University of Goiás) and by a national ethics committee (National Committee for Research Ethics/Brazil). The Nepal Health Research Council, under the Ministry of Health of the Government of Nepal, also provided ethical approval for this study. All study participants provided written, informed consent. This study was conducted under the supervision of the IDEAL Consortium.

\section{RESULTS}

Main characteristics of study participants in Brazil and Nepal - The main characteristics of the four study groups in Brazil and Nepal are summarised in Table I. The total number of participants tested was 363, of which 180 (49.6\%) were tested in Brazil and $183(50.4 \%)$ in $\mathrm{Ne}-$ pal. In both countries, most MB patients were male $(62 \%$ and $80 \%$ in Brazil and Nepal, respectively) and $84 \%$ of EC in Brazil were female. MB and PB LPs in Brazil were older than the corresponding patients in Nepal. Among the four groups tested in Brazil, the median age was 29 years and $40 \%$ of participants were male; in Nepal, the median age was 33 years and $67 \%$ of the participants were male. Among LPs, a visible BCG scar was present in more than $50 \%$ of the Brazilian PB group. At both study sites, the percentage of visible BCG scars was higher in the EC group than in the HHC group. The same percentage of BI-positive MB LPs (78\%) was detected in Brazil and Nepal. The level of grade 1 disability in the MB patient group was comparable in patients in Nepal and Brazil, but higher in PB LPs in Nepal than in Brazil. The level of grade 2 disability was higher in Nepal in PB and MB LPs. In Brazil, the Ridley-Jopling classification was available for all patients enrolled. For Nepalese patients, Ridley-Jopling classification data were unavailable for 25 MB patients (46.3\%) and 22 PB patients (68.7\%).

ML Flow test results with serum and blood - In Brazil, the ML Flow tests performed with whole blood were positive for $84 \%$ of MB patients and $31 \%$ of PB LPs. All HHC and EC patients tested negative with the ML Flow test with whole blood. In Nepal, the ML Flow test with whole blood was positive for $61 \%$ of MB LPs and 3\% of PB LPs. In the control groups, $8 \%$ of HHC and $2 \%$ of EC were positive.

In Brazil, when serum samples were tested with the ML Flow test, $94 \%$ of MB patients, $44 \%$ of PB patients, $31 \%$ of the HHC group and $2 \%$ of the EC group were positive. When Nepalese serum samples were tested, $69 \%$ of MB patients, $9 \%$ of PB patients, $8 \%$ of the HHC group and $2 \%$ of the EC group were positive according to the ML Flow test.

In Brazil, the agreement of the ML Flow test results for blood and serum was greater than $87 \%(\kappa=0.72)$ for all groups with the exception of the HHC, for which $68.6 \%$ agreement $(\kappa=0.32)$ was observed. In Nepal, the

TABLE I

Main characteristics of the multibacillary (MB) and paucibacillary (PB) leprosy patients recruited in Brazil and Nepal

\begin{tabular}{|c|c|c|c|c|c|c|c|c|}
\hline & \multicolumn{2}{|c|}{ MB } & \multicolumn{2}{|c|}{ PB } & \multicolumn{2}{|c|}{$\mathrm{HHC}$} & \multicolumn{2}{|c|}{$\mathrm{EC}$} \\
\hline & $\begin{array}{c}\text { Brazil } \\
(n=50)\end{array}$ & $\begin{array}{l}\text { Nepal } \\
(\mathrm{n}=54)\end{array}$ & $\begin{array}{c}\text { Brazil } \\
(n=45)\end{array}$ & $\begin{array}{l}\text { Nepal } \\
(\mathrm{n}=29)\end{array}$ & $\begin{array}{c}\text { Brazil } \\
(n=35)\end{array}$ & $\begin{array}{l}\text { Nepal } \\
(n=49)\end{array}$ & $\begin{array}{c}\text { Brazil } \\
(n=50)\end{array}$ & $\begin{array}{l}\text { Nepal } \\
(\mathrm{n}=51)\end{array}$ \\
\hline Sex female $[\mathrm{n}(\%)]$ & $19(38)$ & $11(20)$ & $26(58)$ & $10(35)$ & $21(60)$ & $18(37)$ & $42(84)$ & $22(43)$ \\
\hline Age (years, range) ${ }^{a}$ & $48(23-100)$ & $35(16-73)$ & $41(18-76)$ & $33(16-64)$ & $29(18-76)$ & $31(2-60)$ & $20(18-56)$ & $21(17-58)$ \\
\hline BCG scar [n (\%)] & $16(32)$ & $14(29)$ & $24(53)$ & $11(38)$ & $19(54)$ & $26(53)$ & $46(92)$ & $42(88)$ \\
\hline BI positivity [n (\%)] & $39(78)$ & $42(78)$ & $2(4)$ & 0 & NA & NA & NA & NA \\
\hline \multicolumn{9}{|c|}{ Disability grade $[\mathrm{n}(\%)]$} \\
\hline 1 & $15(30)$ & $16(30)$ & $2(4)$ & $6(19)$ & NA & NA & NA & NA \\
\hline 2 & $4(8)$ & $14(26)$ & - & $3(6)$ & - & - & - & - \\
\hline \multicolumn{9}{|l|}{ Ridley-Jopling ${ }^{b}[\mathrm{n}(\%)]$} \\
\hline $\mathrm{TT}$ & 0 & $1(2)$ & $19(42)$ & $1(3)$ & NA & NA & NA & NA \\
\hline BT & 0 & $9(17)$ & $26(58)$ & $13(45)$ & - & - & - & - \\
\hline $\mathrm{BB}$ & $9(18)$ & 0 & 0 & 0 & - & - & - & - \\
\hline $\mathrm{BL}$ & $13(26)$ & $11(20)$ & 0 & 0 & - & - & - & - \\
\hline LL & $28(56)$ & $9(17)$ & 0 & 0 & - & - & - & - \\
\hline
\end{tabular}

$a$ : median (minimum-maximum); $b$ : in Nepal, $25 \mathrm{MB}$ patients (46.3\%) and $22 \mathrm{~PB}$ patients (68.7\%) did not have data for Ridley and Jopling (1966) classification; BB: borderline-boderline; BI: bacilloscopic index; BL: borderline lepromatous; BT: borderline tuberculoid; EC: endemic control; HHC: household contact; LL: lepromatous; NA: not applicable; TT: tuberculoid. 
agreement between the results of the ML Flow tests performed with blood and serum was greater than $90 \%$ for all groups (Table II).

ML ICA test results - In Brazil, there was no difference between the readings after $5 \mathrm{~min}$ or $10 \mathrm{~min}$ for ML ICA tests performed with serum. In Nepal, the results of the ML ICA tests differed at 10 min (data not shown), with a $5 \%$ increase in positivity vs. readings performed at 5 min without effects on specificity. Because the ML ICA test for whole blood did not perform as specified by the manufacturer, these results were not considered in the analysis.

Agreement between the results of ML ICA and $M L$ Flow tests on the serum of LPS - The general agreement between the results of the ML ICA and ML Flow tests on serum samples in Brazil was $86.3 \%(\kappa=0.71)$. In a stratified analysis, the agreement between the results for the MB patient groups was $96 \%(\kappa=0.73)$. The agreement between the ML ICA and ML Flow tests on serum samples in Nepal was $72.6 \%(\kappa=0.46)$. The agreement for the MB patient group was $83.3 \%(\kappa=0.55)$ (Table III).
The usefulness of the lateral flow tests for leprosy classification - The results of the rapid tests were evaluated for their usefulness for PB and MB leprosy classification. Most MB LPs are anti-PGL-I positive, whereas the majority of $\mathrm{PB}$ patients are seronegative. Based on this relationship, the ML Flow test seropositivity would reclassify as MB 54\% (30/56) and 12\% (5/41) of the patients who were previously classified as PB based on lesion counting in Brazil and Nepal, respectively (Table IV). ML Flow test seronegativity would reclassify as PB $3 \%(1 / 39)$ and $17 \%$ (7/42) of the patients who were classified as MB based on lesion counting in Brazil and Nepal, respectively (Table IV). ML ICA test seropositivity would reclassify as MB 34\% (19/56) and 46\% (19/41) of the PB patients in Brazil and Nepal, respectively; ML ICA test seronegativity would reclassify as PB 10\% (4/39) and 8\% $(5 / 42)$ of the patients classified as MB based on lesion counting in Brazil and Nepal, respectively (Table V).

ML Flow and ML ICA test seropositivity according to the Ridley-Jopling classification and the BI classification - The positive rates of the ML Flow and ML ICA

TABLE II

Agreement between ML Flow tests performed with serum and whole blood

\begin{tabular}{|c|c|c|c|c|c|c|c|c|}
\hline & \multicolumn{4}{|c|}{ Brazil } & \multicolumn{4}{|c|}{ Nepal } \\
\hline & $\begin{array}{c}\mathrm{Se}+/ \mathrm{WB}- \\
(\%)\end{array}$ & $\mathrm{Se}-/ \mathrm{WB}+$ & $\begin{array}{c}\text { AGR } \\
(\%)\end{array}$ & $\mathrm{k}$ & $\begin{array}{c}\mathrm{Se}+/ \mathrm{WB}- \\
(\%)\end{array}$ & Se-/WB+ & $\begin{array}{c}\text { AGR } \\
(\%)\end{array}$ & $\mathrm{k}$ \\
\hline MB & 10 & 0 & 90 & 0.50 & 7.4 & 0 & 92.6 & 0.83 \\
\hline PB & 12.5 & 0 & 87.5 & 0.73 & - & 0 & 93.8 & 0.47 \\
\hline HHC & 31.4 & 0 & 68.6 & 0.32 & 4.1 & 4.1 & 91.8 & 0.45 \\
\hline $\mathrm{EC}$ & 2 & 0 & 98 & 0.85 & 0 & 0 & 100 & 1 \\
\hline Total & 12.6 & 0 & 87.4 & 0.72 & 4.3 & 1.1 & 94.6 & 0.54 \\
\hline
\end{tabular}

AGR: agreement; EC: endemic control; HHC: household contact; k: kappa value; MB: multibacillary; PB: paucibacillary; Se: serum; WB: whole blood; +: positive; -: negative.

TABLE III

Agreement between ML ICA and ML Flow tests with serum in Nepal and Brazil

\begin{tabular}{|c|c|c|c|c|c|c|c|c|}
\hline & \multicolumn{4}{|c|}{ Brazil } & \multicolumn{4}{|c|}{ Nepal } \\
\hline & $\begin{array}{c}\text { Flow }+/ \text { ICA- } \\
(\%)\end{array}$ & $\begin{array}{c}\text { Flow-/ICA }+ \\
(\%)\end{array}$ & $\begin{array}{c}\text { AGR } \\
(\%)\end{array}$ & $\mathrm{k}$ & $\begin{array}{c}\text { Flow }+/ \text { ICA- } \\
(\%)\end{array}$ & $\begin{array}{c}\text { Flow-/ICA }+ \\
(\%)\end{array}$ & $\begin{array}{l}\text { AGR } \\
(\%)\end{array}$ & $\mathrm{k}$ \\
\hline MB & 4 & 0 & 96 & 0.73 & 0 & 14.8 & 83.3 & 0.55 \\
\hline PB & 27.1 & 2.1 & 70.8 & 0.36 & 0 & 28.1 & 68.8 & 0.26 \\
\hline $\mathrm{HHC}$ & 17.1 & 5.7 & 77.1 & 0.41 & 0 & 26.5 & 59.2 & 0.17 \\
\hline EC & 2 & 0 & 98 & 0.86 & 0 & 23.5 & 76.5 & 0.11 \\
\hline Total & 12 & 1.6 & 86.3 & 0.71 & 0 & 22.6 & 72.6 & 0.46 \\
\hline
\end{tabular}

readings of ML Flow tests were performed at 10 min. AGR: agreement; EC: endemic control; HHC: household contact; k: kappa value; MB: multibacillary; PB: paucibacillary; +: positive; -: negative. 
tests were higher in LL patients than in TT patients (Table VI). Table VII shows that the seropositivity rate of the ML Flow and ML ICA tests increased with increasing patient BI values in Brazil and Nepal.

\section{DISCUSSION}

Serological studies of leprosy suggest that the detection of anti-PGL-I antibodies, although specific, should not be used for diagnosis, but may be useful for the classification of MB and PB forms of leprosy (Bührer-Sékula et al. 2003, Oskam et al. 2003). Anti-PGL-I testing is usually based on the detection of IgM antibodies, but the detection of anti-PGL-I IgG and IgA antibodies has also been described (Cho et al. 1984, Saad et al. 1991). The first rapid anti-PGL-I test (ML Flow test) was developed based on the detection of IgM antibodies, but has low sensitivity in PB leprosy (Bührer-Sékula et al. 2003). In an attempt to improve the sensitivity of the rapid anti-PGL-I test, another format, called ML ICA, was developed that simultaneously detects $\operatorname{IgM}, \operatorname{IgG}$ and IgA antibodies. This study compared the results of the ML Flow and ML ICA tests and evaluated the applicability of using serological data as an additional tool for the classification of PB and MB leprosy.

In an intra-test comparison, there were no statistically significant differences among any of the groups when the ML Flow test was used with whole blood or serum, indicating that both whole blood and serum can be used. These results are consistent with previous studies that showed a very strong correlation between the results from whole blood and serum (Bührer-Sékula et al. 2003).

Because the ML ICA test for whole blood did not perform according to the manufacturer's specifications, we were only able to use the serum results. With the ML ICA tests performed in Nepal, we observed different results when the tests were read at $5 \mathrm{~min}$ and $10 \mathrm{~min}$. Ap- proximately an additional $5 \%$ of the tests became positive at the 10 min reading, with no effects on test specificity. However, the correlation between the readings at $5 \mathrm{~min}$ and $10 \mathrm{~min}$ was high. When taking all groups into account, the agreement was very good $(94 \% ; \mathrm{k}=0.88)$. Among MB LPs, the correlation between the two readings was even higher, with $98.1 \%$ agreement $(\mathrm{k}=0.95)$. Due to the higher positive rate among MB patients at the 10 min reading, we recommend using this time for ML ICA tests. When we compared the ML ICA test results at $10 \mathrm{~min}$, the correlation among all groups in Brazil and Nepal was $97 \%(\mathrm{k}=0.94)$. Again, the highest correlation was observed in the MB patient groups $(99 \%, \mathrm{k}=0.96)$.

The inter-test comparison between the ML ICA and ML Flow tests with serum samples showed good agreement in Brazil $(86.3 \%$; $\mathrm{k}=0.71)$, but the agreement was classified as moderate in Nepal $(72.6 \% ; \mathrm{k}=0.46)$. In a stratified analysis by study group, only the results for MB patients from Brazil and Nepal and for EC from Brazil exhibited either very good or good agreement. The fair agreement observed with test results for PB patients and HHC from Brazil and Nepal suggest that (i) the higher positive rate in the ML Flow test in Brazil for most groups is likely the result of non-specific binding in the ML Flow test and (ii) the higher positive response of the ML ICA test for all groups in Nepal is most likely due to the detection of IgG and IgA antibodies. The high agreement between the two tests for the EC group in Brazil may be the result of chance equilibrium between non-specific binding in the ML Flow test and the detection of IgG and IgA antibodies by the ML ICA test. This possibility is supported by the fact that, in Nepal, none of samples that were positive in the ML Flow test were negative by the ML ICA test and $22.6 \%$ of ML Flow test negative samples for all study populations were positive in the ML ICA test (Table III).

TABLE IV

Comparison between classifications based on the number

of skin lesions and results of ML Flow and smear examination for Brazil and Nepal

\begin{tabular}{|c|c|c|c|c|c|}
\hline \multirow{2}{*}{$\begin{array}{l}\text { Classification based on } \\
\text { WHO lesion counting }\end{array}$} & \multicolumn{2}{|c|}{ Confirmatory tests } & \multirow{2}{*}{$\begin{array}{l}\text { Brazil } \\
\mathrm{n}(\%)\end{array}$} & \multirow{2}{*}{$\begin{array}{l}\text { Nepal } \\
\text { n }(\%)\end{array}$} & \\
\hline & ML Flow & Smear & & & \\
\hline \multirow[t]{4}{*}{ PB patients } & + & + & $10(18)$ & $2(5)$ & \multirow{2}{*}{ Misclassified $^{a}$} \\
\hline & - & + & $3(5)$ & $6(15)$ & \\
\hline & + & - & $20(36)$ & $3(7)$ & \multirow{2}{*}{ Confirmed PB } \\
\hline & - & - & $23(41)$ & $30(73)$ & \\
\hline Total & & & $56(59)$ & $41(49)$ & \\
\hline \multirow[t]{4}{*}{ MB patients } & + & + & $28(72)$ & $31(74)$ & \multirow{2}{*}{ Confirmed $\mathrm{MB}^{a}$} \\
\hline & - & + & $0(0)$ & $3(7)$ & \\
\hline & + & - & $10(26)$ & $4(10)$ & \multirow{2}{*}{ Misclassified? } \\
\hline & - & - & $1(2)$ & $4(9)$ & \\
\hline Total & & & $39(41)$ & $42(51)$ & \\
\hline
\end{tabular}

$a$ : based on smear positivity; MB: multibacillary; PB: paucibacillary; +: positive; -: negative. 
The high agreement between the tests for the MB group and the low agreement among other groups could be clarified by an analysis of the patients' operational classification based on number of skin lesions. The BI positivity for MB patients based on the number of lesions was $72 \%$ and $81 \%$ in Brazil and in Nepal, respectively, which led to high positivity for both serological tests. The ML Flow test confirmed the MB classification by lesion number for $72 \%$ and $74 \%$ of patients in Brazil and in Nepal, respectively and the ML ICA test confirmed the MB classification for $67 \%$ of patients in Brazil and $76 \%$ of patients in Nepal.

Differences were observed among PB LPs classified based on the number of lesions. In Brazil, PB patients with five or fewer skin lesions had a higher BI positivity rate (24\%) than patients in Nepal $(19 \%)$. Based on this difference, we expected a lower positive rate of serological tests in Nepal and indeed, we observed an ML Flow test positivity rate of $12 \%$ for PB patients in Nepal, compared with $54 \%$ in Brazil ( $\mathrm{p}=0.00002)$. By contrast, $47 \%$ of $\mathrm{PB}$ patients in Nepal were positive according to the ML ICA test, compared to $34 \%$ of PB patients in Brazil. In addition, among the BI-negative PB LPs from Nepal, the ML ICA test had a significantly higher positive rate, $30 \%$, compared to $7 \%$ when using the ML Flow test $(p=0.0005)$. The negative results of the ML Flow test confirmed the classification of $41 \%$ of PB patients in Brazil and $73 \%$ in Nepal; the ML ICA test confirmed the PB classification of $62 \%$ and $51 \%$ of PB patients in Brazil and Nepal, respectively. The higher positive rate of the PB group in Brazil and the lower positive rate of PB patients in Nepal of the ML Flow test may be partially explained by the diversity in the ratios of leprosy type among these populations, particularly when considering BT-MB cases. As shown in Table I, whereas some BT patients in Nepal were classified as MB, there were no such cases in Brazil. Patients in Nepal also had higher disability scores than those in Brazil, which may indicate different severities of $M$. leprae infection among these groups. In addition, it is also important to consider that these are relatively small samples of large populations from two different endemic populations.

Nevertheless, one must also consider the possible effects of the different carrier proteins used for the PGL-I carbohydrate portion of the neoglycoprotein antigens. The ML ICA test uses both BSA and HSA, while the ML Flow test uses only BSA, a protein to which humans can produce antibodies, which may lead to non-specific binding and interfere with the test results. The higher positive rate of the ML ICA test among PB patients in Brazil and Nepal compared to the ML Flow test may be due to the detection of specific $\operatorname{IgA}$ and $\operatorname{IgG}$ antibodies to PGL-I, as has been observed in previous studies (Chujor et al. 1991).

Overall, our results indicate that the addition of anti-IgG and anti-IgA antibody detection in the ML ICA test did not improve the sensitivity of the test sufficiently to warrant its use as a diagnostic test. Moreover, if the goal of the test is to aid in the classification of PB and MB leprosy, it may be advantageous to detect only anti-PGL-I IgM antibodies as these results more clearly differentiate between PB and MB leprosy. This distinction is important for treatment purposes. As observed in Nepal, when considering the number of lesions, the performance of the ML Flow test was superior: seropositivity confirmed the correct classification of $74 \%$ of MB patients and seronegativity confirmed the operational classification of $73 \%$ of PB patients. These results indicate that the anti-PGL-I ML Flow test can be a useful tool for the correct classification of MB and PB LPs in

TABLE V

Comparison between classifications based on the number of skin lesions and results of ML ICA and smear examination for Brazil and Nepal

\begin{tabular}{|c|c|c|c|c|c|}
\hline \multirow{2}{*}{$\begin{array}{l}\text { Classification based on } \\
\text { WHO lesion counting }\end{array}$} & \multicolumn{2}{|c|}{ Confirmatory tests } & \multirow{2}{*}{$\begin{array}{l}\text { Brazil } \\
\text { n (\%) }\end{array}$} & \multirow{2}{*}{$\begin{array}{l}\text { Nepal } \\
\text { n (\%) }\end{array}$} & \\
\hline & ML ICA & Smear & & & \\
\hline \multirow[t]{4}{*}{ PB patients } & + & + & $11(20)$ & $7(17)$ & \multirow{2}{*}{ Misclassified $^{a}$} \\
\hline & - & + & $2(4)$ & $1(2)$ & \\
\hline & + & - & $8(14)$ & $12(30)$ & \multirow{2}{*}{ Confirmed PB } \\
\hline & - & - & $35(62)$ & $21(51)$ & \\
\hline Total PB & & & $56(59)$ & $41(49)$ & \\
\hline \multirow[t]{4}{*}{ MB patients } & + & + & $26(67)$ & $32(76)$ & \multirow{2}{*}{ Confirmed $\mathrm{MB}^{a}$} \\
\hline & - & + & $2(5)$ & $2(5)$ & \\
\hline & + & - & $9(23)$ & $5(12)$ & \multirow{2}{*}{ Misclassified? } \\
\hline & - & - & $2(5)$ & $3(7)$ & \\
\hline Total MB & & & $39(41)$ & $42(51)$ & \\
\hline
\end{tabular}

$a$ : based on smear positivity; MB: multibacillary; PB: paucibacillary; +: positive; -: negative. 
this population to facilitate better treatment decisions. This type of additional testing may help identify those clinically diagnosed (by lesion counting or negative $\mathrm{BI}$ ) PB patients that should be reconsidered for MB treatment. Clinicians lacking access to smears could use antiPGL-I serology to further test uncertain PB cases before assigning treatment.

In the Brazilian subjects, the ML Flow test confirmed the correct classification of $41 \%$ of PB patients and $72 \%$ of MB patients. This lower confirmation rate for PB leprosy may indicate that using HSA instead of BSA as the neoglycolipid carrier in the ML Flow test could enhance the specificity of the test.

From an operational standpoint, the ML Flow test format has advantages over the ML ICA format because a single test format can be used for both whole blood and serum samples. In addition, the ML Flow test comes in individual packages, while the ML ICA test comes in packs of 10 cassettes each; once opened, the stability of unused tests may be impaired. Moreover, from a practical perspective, the ML ICA test is slightly more difficult to implement because it requires prior sample serum dilution. Nevertheless, the format of any test can be optimised to fulfil the needs of the end users, who often work in peripheral health clinics with limited facilities. Because previous serum dilution is not necessary for the ML Flow test, it is the easiest test to perform in non-specialised settings. Considering the need for a rapid test for use in field situations in endemic countries, the use of whole blood samples offers obvious advantag-

\section{TABLE VI}

Comparison between Ridley-Jopling classification and results of ML Flow and ML ICA for Brazil and Nepal

\begin{tabular}{lcccc}
\hline & \multicolumn{2}{c}{$\begin{array}{c}\text { Brazil } \\
(\mathrm{n}=95)\end{array}$} & \multicolumn{2}{c}{$\begin{array}{c}\text { Nepal } \\
(\mathrm{n}=44)\end{array}$} \\
\cline { 2 - 4 } \cline { 5 - 6 } & $\begin{array}{c}\text { Positive } \\
\mathrm{n}(\%)\end{array}$ & $\begin{array}{c}\text { Negative } \\
\mathrm{n}(\%)\end{array}$ & $\begin{array}{c}\text { Positive } \\
\mathrm{n}(\%)\end{array}$ & $\begin{array}{c}\text { Negative } \\
\mathrm{n}(\%)\end{array}$ \\
\hline Ridley-Jopling ${ }^{a}$ & \multicolumn{4}{c}{ ML Flow } \\
TT & $10(53)$ & $9(47)$ & $0(0)$ & $2(100)$ \\
BT & $12(46)$ & $14(54)$ & $9(41)$ & $13(59)$ \\
BB & $6(67)$ & $3(33)$ & $0(0)$ & - \\
BL & $12(92)$ & $1(8)$ & $10(91)$ & $1(9)$ \\
LL & $28(100)$ & - & $8(100)$ & - \\
& \multicolumn{5}{c}{ ML ICA } \\
TT & $4(21)$ & $15(79)$ & $0(0)$ & $2(100)$ \\
BT & $6(23)$ & $20(77)$ & $14(61)$ & $9(39)$ \\
BB & $5(55)$ & $4(45)$ & $0(0)$ & - \\
BL & $11(85)$ & $2(15)$ & $11(100)$ & - \\
LL & $28(100)$ & - & $8(100)$ & -
\end{tabular}

$a$ : Ridley and Jopling (1966) classification; BB: borderlineboderline; BL: borderline lepromatous; BT: borderline tuberculoid; LL: lepromatous; TT: tuberculoid. es because it is easier to obtain, such as by finger prick at the time of consultation, requires less handling and can be performed at the POC facility (Bührer-Sékula et al. 1998). However, the testing of serum did result in a significant gain in sensitivity at both sites and could be used for additional or repeat testing if required.

Although the Ridley-Jopling classification is considered the gold standard for research purposes, this classification system is complex and involves clinical, microbiological and histopathological criteria. In this study, a standardised protocol for Ridley-Jopling classification was not used for patients in Nepal and Brazil; therefore, we cannot exclude discrepancies due to possible misclassifications. Moreover, due to operational constraints, the Ridley-Jopling classification was available for only approximately half of the Nepalese patients, which limited the analysis. The WHO operational classification for MB and PB leprosy (skin lesion counting) also has shortcomings, but it has been considered useful in lowincome leprosy endemic countries. The classification of MB and PB leprosy based on anti PGL-I serology also has limitations because not all MB patients are seropositive. In this regard, one of the limitations of the lateral flow test is that visual readings may lead to a reading bias towards MB positivity and PB negativity if they are not blinded from the clinical classification. Therefore, we cannot exclude the possibility that operational procedures may have had an impact on the POC test results. When considering the Ridley-Jopling classification,

\section{TABLE VII}

Comparison between results of bacterial index (BI), ML Flow and ML ICA for Brazil and Nepal

\begin{tabular}{|c|c|c|c|c|}
\hline & \multicolumn{2}{|c|}{$\begin{array}{c}\text { Brazil } \\
(\mathrm{n}=95)\end{array}$} & \multicolumn{2}{|c|}{$\begin{array}{c}\text { Nepal } \\
(\mathrm{n}=83)\end{array}$} \\
\hline & $\begin{array}{c}\text { Positive } \\
\text { n (\%) }\end{array}$ & $\begin{array}{c}\text { Negative } \\
\mathrm{n}(\%)\end{array}$ & $\begin{array}{c}\text { Positive } \\
\text { n (\%) }\end{array}$ & $\begin{array}{c}\text { Negative } \\
\text { n }(\%)\end{array}$ \\
\hline BI & \multicolumn{4}{|c|}{ ML Flow } \\
\hline 0 & $30(56)$ & $24(44)$ & 7 (17) & $34(83)$ \\
\hline 1 & $8(80)$ & $2(20)$ & $6(55)$ & $5(45)$ \\
\hline 2 & $8(89)$ & $1(11)$ & $3(60)$ & $2(40)$ \\
\hline 3 & $9(100)$ & - & $6(86)$ & $1(14)$ \\
\hline 4 & $9(100)$ & - & $15(94)$ & $1(6)$ \\
\hline 5 & $3(100)$ & - & $3(100)$ & - \\
\hline \multirow[t]{2}{*}{6} & $1(100)$ & - & - & - \\
\hline & \multicolumn{4}{|c|}{ ML ICA } \\
\hline 0 & $17(31)$ & 37 (69) & $17(41)$ & $24(59)$ \\
\hline 1 & $9(90)$ & $1(10)$ & $10(91)$ & $1(9)$ \\
\hline 2 & $8(89)$ & $1(11)$ & $4(80)$ & $1(20)$ \\
\hline 3 & $8(89)$ & $1(11)$ & $7(100)$ & - \\
\hline 4 & $9(100)$ & - & $15(94)$ & $1(6)$ \\
\hline 5 & $2(67)$ & $1(33)$ & $3(100)$ & - \\
\hline 6 & $1(100)$ & - & - & - \\
\hline
\end{tabular}


the positive rate of the ML Flow test was higher in LL patients than in TT patients (56\% vs. $0 \%$ ) (Table VI), which most likely reflects the increase in the bacillary load of the patients in Brazil. Only newly diagnosed, untreated Brazilian patients were enrolled. The RidleyJopling classification could not have interfered with the readings because the histopathology and $\mathrm{BI}$ results were not available at the POC facility when the patients were diagnosed and tested. Despite the limitations of serology for diagnosing or classifying leprosy, the identification of MB patients by serology will remain important: MB patients are the main source of transmission and after MDT they present a higher risk for relapse and leprosyrelated reactions, which are the main cause of deformities and incapacities. Therefore, any tool that may aid the identification of MB LPs with a higher risk for complications may contribute to improved patient management and leprosy control in endemic countries.

Our results demonstrate that when the different antiPGL-I POC tests were performed to facilitate the operational classification of PB and MB leprosy for treatment purposes, the ML Flow test was the best POC test for patients from Nepal. Despite the need for sample dilution, the ML ICA test gave better results than the ML Flow test in the Brazilian group. Our results identified possible ways to improve the performance of both tests. As a POC test to classify patients as $\mathrm{PB}$ or MB for treatment purposes, anti PGL-I serology should involve the use of undiluted whole blood samples to detect IgM antibodies to the neoglycolipid conjugated to the HSA antigen carrier.

\section{ACKNOWLEDGEMENTS}

To all participants of this study, to the support of the participating sites, lab and clinical staff of Anandaban Hospital, Anandaban's weekly satellite clinic at Patan Hospital in Kathamandu, Nepal, and from Reference Center for Diagnostics and Therapeutics, Goiânia, state of Goiás, Brazil, and to Dr Tsuyoshi Fujiwara, for providing the NT-P-BSA antigen used in the ML Flow test.

\section{REFERENCES}

Brett SJ, Payne SN, Gigg J, Burgess P, Gigg R 1986. Use of synthetic glycoconjugates containing the Mycobacterium leprae specific and immunodominant epitope of phenolic glycolipid-I in the serology of leprosy. Clin Exp Immunol 64: 476-483.

Bührer-Sékula S, Illarramendi X, Teles RB, Penna ML, Nery JA, Sales AM, Oskam L, Sampaio EP, Sarno EN 2009. The additional benefit of the ML Flow test to classify leprosy patients. Acta Trop 111: 172-176.

Bührer-Sékula S, Smits HL, Gussenhoven GC, van Ingen CW, Klatser PR 1998. A simple dipstick assay for the detection of antibodies to phenolic glycolipid-I of Mycobacterium leprae. Am J Trop Med Hyg 58: 133-136.

Bührer-Sékula S, Smits HL, Gussenhoven GC, van Leeuwen J, Amador S, Fujiwara T, Klatser PR, Oskam L 2003. Simple and fast lateral flow test for classification of leprosy patients and identi- fication of contacts with high risk of developing leprosy. J Clin Microbiol 41: 1991-1995.

Chanteau S, Cartel JL, Celerier P, Plichart R, Desforges S, Roux J 1989. PGL-I antigen and antibody detection in leprosy patients: evolution under chemotherapy. Int J Lepr Other Mycobact Dis 57: 735-743.

Cho SN, Cellona RV, Fajardo Jr TT, Abalos RM, la Cruz EC, Walsh GP, Kim JD, Brennan PJ 1991. Detection of phenolic glycolipid-I antigen and antibody in sera from new and relapsed lepromatous patients treated with various drug regimens. Int J Lepr Other Mycobact Dis 59: 25-31.

Cho SN, Fujiwara T, Hunter SW, Rea TH, Gelber RH, Brennan PJ 1984. Use of an artificial antigen containing the 3,6-di-O-methylbeta-D-glucopyranosyl epitope for the serodiagnosis of leprosy. J Infect Dis 150: 311-322.

Chujor CS, Bernheimer H, Levis WR, Schwerer B 1991. Serum IgA1 and IgM antibodies against Mycobacterium leprae-derived phenolic glycolipid-I: a comparative study in leprosy patients and their contacts. Int J Lepr Other Mycobact Dis 59: 441-449.

Dockrell HM, Geluk A, Brennan P, Saunderson PR, Oskam L, Lockwood DN, Xiaoman W, Richardus JH 2011. Report on the sixth meeting of the IDEAL (Initiative for Diagnostic and Epidemiological Assays for Leprosy) consortium held in Beijing, China on 23-25 August 2010. Lepr Rev 82: 80-85.

Douglas JT, Worth RM 1984. Field evaluation of an ELISA to detect antibody in leprosy patients and their contacts. Int J Lepr Other Mycobact Dis 52: 26-33.

Moura RS, Calado KL, Oliveira ML, Bührer-Sékula S 2008. Leprosy serology using PGL-I: a systematic review. Rev Soc Bras Med Trop 41 (Suppl.): 11-18.

Oskam L, Slim E, Bührer-Sékula S 2003. Serology: recent developments, strengths, limitations and prospects: a state of the art overview. Lepr Rev 74: 196-205.

Petchclai B, Khupulsup K, Hiranras S, Sampatavanich S, Sampoonachot $P$, Leelarusamee A 1988. A passive hemagglutination test for leprosy using a synthetic disaccharide antigen. Int J Lepr Other Mycobact Dis 56: 255-258.

Ridley DS, Jopling WH 1966. Classification of leprosy according to immunity. A five-group system. Int J Lepr Other Mycobact Dis 34: 255-273.

Saad MH, Medeiros MA, Gallo ME, Gontijo-Filho PP, Fonseca LS 1991. The dot-ELISA test for the detection of anti-PGL-1 IgM in leprosy patients and their contacts. Braz J Med Biol Res 24: 441-448.

Sampaio LH, Stefani MM, Oliveira RM, Sousa AL, Ireton GC, Reed SG, Duthie MS 2011. Immunologically reactive $M$. leprae antigens with relevance to diagnosis and vaccine development. $B M C$ Infect Dis 11: 26.

Scollard DM 2008. The biology of nerve injury in leprosy. Lepr Rev 79: $242-253$.

WHO - World Health Organization 1988. WHO Expert Committee on Leprosy, Sixth Report. WHO Tech Rep Ser 874: 1-143.

WHO - World Health Organization 1998. WHO Expert Committee on Leprosy, WHO, Geneva, 73 pp.

WHO - World Health Organization 2010. Global leprosy situation 2010. Wkly Epidemiol Rec 85: 337-348. 